\title{
MASCOT: an ESO-ARO legacy survey of molecular gas in nearby SDSS-MaNGA galaxies - I. First data release, and global and resolved relations between $\mathrm{H}_{2}$ and stellar content
}

\author{
D. Wylezalek ${ }^{\oplus,}{ }^{1 \star}$ C. Cicone, ${ }^{2}$ F. Belfiore ${ }^{\oplus},{ }^{3}$ C. Bertemes,${ }^{1}$ S. Cazzoli ${ }^{\oplus},{ }^{4}$ J. Wagg, ${ }^{5}$
}

W. Wang (王无忌) ${ }^{\oplus},{ }^{1}$ M. Aravena, ${ }^{6}$ R. Maiolino, ${ }^{7,8}$ S. Martin,,${ }^{9,10}$ M. S. Bothwell, ${ }^{7,8}$ J. R. Brownstein ${ }^{\circledR}, 11$

K. Bundy ${ }^{12,13}$ and C. De Breuck ${ }^{14}$

${ }^{1}$ Zentrum für Astronomie der Universität Heidelberg, Astronomisches Rechen-Institut, Mönchhofstr 12-14, D-69120 Heidelberg, Germany

${ }^{2}$ Institute of Theoretical Astrophysics, University of Oslo, PO Box 1029, Blindern, 0315 Oslo, Norway

${ }^{3}$ Osservatorio Astrofisico di Arcetri, INAF, Largo Enrico Fermi 5, I-50125 Firenze, Italy

${ }^{4}$ IAA - Instituto de Astrofísica de Andalucía, CSIC, Apdo. 3004, E-18008 Granada, Spain

${ }^{5}$ SKA Observatory, Lower Withington Macclesfield, Cheshire SK11 9FT, UK

${ }^{6}$ Núcleo de Astronomía, Facultad de Ingeniería y Ciencias, Universidad Diego Portales, Av. Ejército 441, Santiago, 8320000, Chile

${ }^{7}$ Cavendish Laboratory, University of Cambridge, Cambridge CB3 OHE, UK

${ }^{8}$ Kavli Institute for Cosmology, University of Cambridge, Cambridge CB3 OHE, UK

${ }^{9}$ European Southern Observatory, Alonso de Córdova, 3107 Vitacura, Santiago 763-0355, Chile

${ }^{10}$ Joint ALMA Observatory, Alonso de Córdova, 3107 Vitacura, Santiago 763-0355, Chile

${ }^{11}$ Department of Physics and Astronomy, University of Utah, 115 S. 1400 E., Salt Lake City, UT 84112, USA

${ }^{12}$ Department of Astronomy and Astrophysics, University of California, 1156 High Street, Santa Cruz, CA 95064, USA

${ }^{13}$ UCO/Lick Observatory, Department of Astronomy and Astrophysics, University of California, 1156 High Street, Santa Cruz, CA 95064, USA

${ }^{14}$ European Southern Observatory, Karl-Schwarzschild-Str 2, D-85748 Garching, Germany

Accepted 2021 November 11. Received 2021 November 11; in original form 2021 August 2

\begin{abstract}
We present the first data release of the Mapping Nearby Galaxies at Apache Point Observatory (MaNGA)-Arizona Radio Observatory (ARO) Survey of CO Targets (MASCOT), a European Southern Observatory public spectroscopic survey conducted at the ARO. We measure the $\mathrm{CO}(1-0)$ line emission in a sample of 187 nearby galaxies selected from the MaNGA survey that has obtained integral field unit (IFU) spectroscopy for a sample of $\sim 10000$ galaxies at low redshift. The main goal of MASCOT is to probe the molecular gas content of star-forming galaxies with stellar masses $>10^{9.5} \mathrm{M}_{\odot}$ and with associated MaNGA IFU observations and well-constrained quantities like stellar masses, star formation rates, and metallicities. In this paper, we present the first results of the MASCOT survey, providing integrated $\mathrm{CO}(1-0)$ measurements that cover several effective radii of the galaxy and present $\mathrm{CO}$ luminosities, $\mathrm{CO}$ kinematics, and estimated $\mathrm{H}_{2}$ gas masses. We observe that the decline of the galaxy star formation rate with respect to the star formation main sequence increases with the decrease of molecular gas and with a reduced star formation efficiency, in agreement with results of other integrated studies. Relating the molecular gas mass fractions with the slope of the stellar age gradients inferred from the MaNGA observations, we find that galaxies with lower molecular gas mass fractions tend to show older stellar populations close to the galactic centre, while the opposite is true for galaxies with higher molecular gas mass fractions, providing tentative evidence for inside-out quenching.
\end{abstract}

Key words: surveys -ISM: general-galaxies: evolution-galaxies: ISM.

\section{INTRODUCTION}

Understanding the behaviour of the cold phase of the interstellar medium (ISM) is central to understanding the galaxy evolution process as a whole. Galaxies exist in a state of flux, being subject to a range of physical processes (including accretion, gas outflow, and star formation) that are the drivers of their evolution (Tumlinson, Peeples \& Werk 2017; Péroux \& Howk 2020). For example, star formation is regulated by the amount of available gas and internal

^E-mail: dominika.wylezalek@uni-heidelberg.de feedback processes (Kennicutt 1998b; Harrison 2017; Rupke 2018). The tight relation between molecular gas and star formation has been studied for decades (Sanders \& Mirabel 1985; Solomon et al. 1997; Combes, Young \& Bureau 2007; Young et al. 2011; Saintonge et al. 2011, 2017; Lin et al. 2019b; Colombo et al. 2020), and has come to represent a central pillar of our understanding of galaxy evolution as well as a critical ingredient in models (Bouché et al. 2010; Lilly et al. 2013; Saintonge et al. 2016). However, disentangling the detailed physics underlying this correlation remains an important area of research. As such, attaining a better understanding of the gas content of galaxies, in particular the molecular gas content, which is the fuel for star formation, is key to understanding how galaxies evolve. 


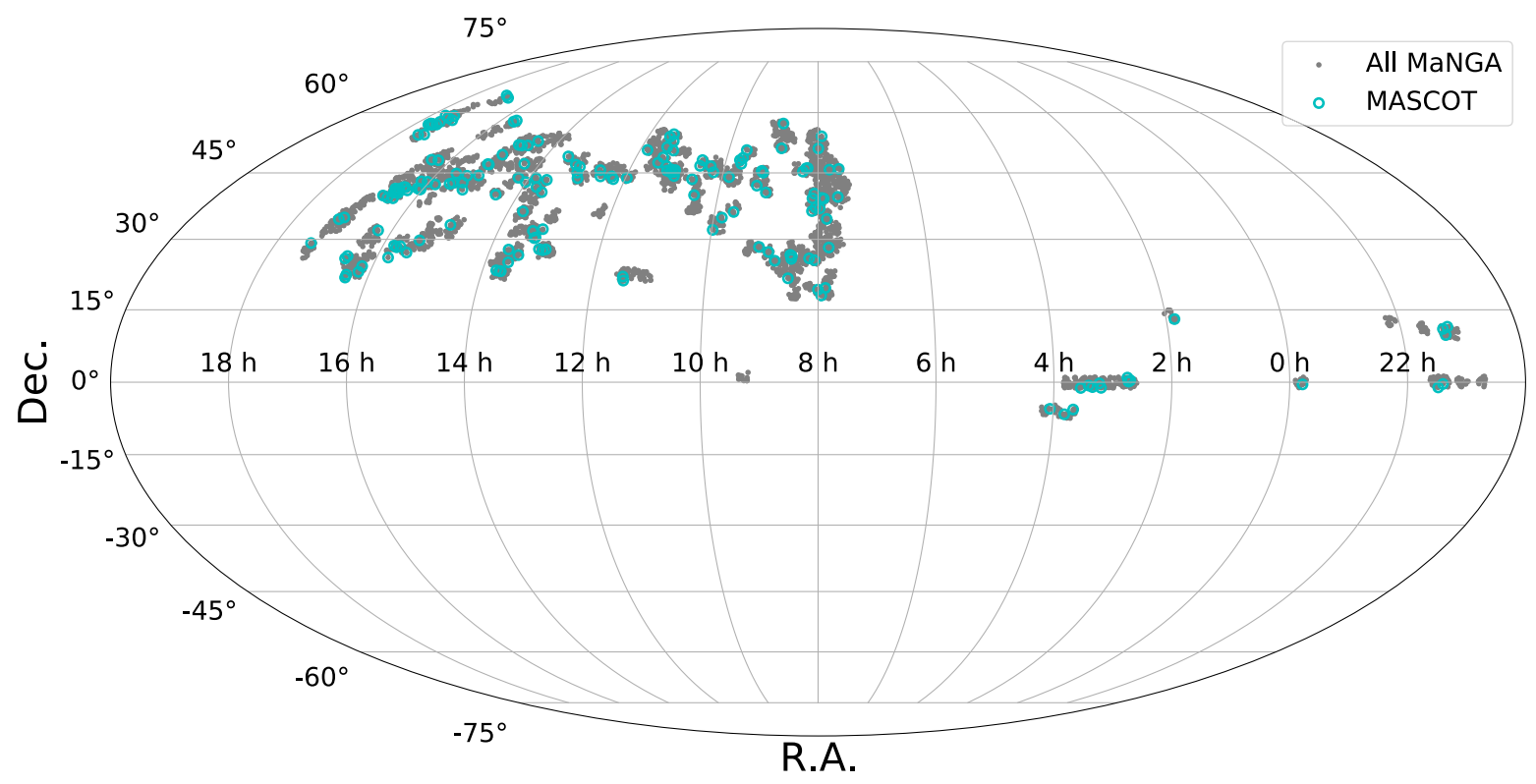

Figure 1. Sky distribution of observed MASCOT sources. We also show the distribution of the DR15 MaNGA sample as small grey points.

Carbon monoxide, ${ }^{12} \mathrm{C}^{16} \mathrm{O}$, is the most widely used tracer of molecular gas, both in the local and in the high- $z$ Universe. The luminosities of the low- $J$ CO transitions, in particular the lowest energy $\mathrm{CO}(1-0)$ emission line (rest frequency $v=115.271 \mathrm{GHz}$ ), can be used to measure the mass of molecular gas, modulo a conversion factor $\alpha_{\mathrm{CO}}$ (for a review, see Bolatto, Wolfire \& Leroy 2013). Several large surveys have therefore targeted the low- $J$ transitions of $\mathrm{CO}$ to measure the probe gas content in the past decade. The CO Legacy Database for GALEX Arecibo SDSS Survey (GASS) (COLD GASS; Saintonge et al. 2011) survey used the IRAM $30 \mathrm{~m}$ telescope to measure the $\mathrm{CO}(1-0)$ line in a sample of $\sim 350$ nearby $(D=100$ $200 \mathrm{Mpc})$ massive galaxies with $\log \left(M_{*} / \mathrm{M}_{\odot}\right)>10.0$ selected from the Sloan Digital Sky Survey (SDSS). The survey was later expanded to also include lower mass galaxies (xCOLD GASS; Saintonge et al. 2017), bringing the combined surveys to a sample size of 532 galaxies probing the entire star formation rate (SFR) and stellar mass plane above $\log \left(M_{*} / \mathrm{M}_{\odot}\right)>9.0$. Furthermore, the Atacama Pathfinder Experiment (APEX) Low-redshift Legacy Survey for MOlecular Gas (ALLSMOG; Bothwell et al. 2014; Cicone et al. 2017) complements the $\mathrm{xCOLD}$ GASS survey by providing $\mathrm{CO}(2-1)$ emission-line observations of 88 nearby low-mass $\left[8.5<\log \left(M_{*} / \mathrm{M}_{\odot}\right)<10\right]$ galaxies taken with the APEX-1 receiver on the APEX telescope. These surveys revealed that it is both the molecular gas mass fraction, i.e. the molecular gas to stellar mass ratio $f_{\mathrm{H}_{2}}=M_{\mathrm{H}_{2}} / M_{*}$, and the star formation efficiency ( $\mathrm{SFE}=\mathrm{SFR} / M_{\mathrm{H}_{2}}$ ) that vary strongly as a function of specific SFR and determine a galaxy's offset from the star-forming main sequence. The CO luminosity not only appears to correlate strongly with stellar mass and SFR, but also varies with metallicity and $\mathrm{HI}$ mass.

These surveys were able to explore the connection between the global gas content of galaxies where galaxy parameters had been obtained via single-fibre SDSS spectroscopy (and SDSS photometry). That means all information about stellar mass, SFR, central active galactic nucleus (AGN) activity, concentration parameter, and metallicity was therefore restricted to a central 3 arcsec aperture probing very different spatial scales depending on the redshift of the source. Integral field unit (IFU) surveys now offer new possibilities in investigating the spatial dimension of galaxy evolution and allow these quantities to be mapped in two dimensions (Cappellari et al. 2011; Croom et al. 2012; Sánchez et al. 2012; Bundy et al. 2015).

The SDSS-IV (Blanton et al. 2017) survey Mapping Nearby Galaxies at Apache Point Observatory (MaNGA; Bundy et al. 2015; Drory et al. 2015; Law et al. 2015; Yan et al. 2016a,b; Wake et al. 2017) is an optical fibre bundle IFU survey that has mapped the detailed composition and kinematic structure of 10010 unique nearby galaxies at $0.01<z<0.15$. The current public Data Release (DR15) (Aguado et al. 2019) includes data for 4824 MaNGA data cubes. The full MaNGA data set will be released as part of Data Release (DR17), expected in December 2021. MaNGA delivers resolved optical spectroscopic data. Many critical diagnostics which provide insight into the formation processes of galaxies, such as metallicity gradients, age gradients, and resolved AGN diagnostics, are only available to such resolved observations. However, the cold molecular gas phase is not probed by MaNGA observations.

We started to fill in this gap with the MaNGA Survey of CO Targets (MASCOT; Fig. 1). MASCOT was initially granted $200 \mathrm{~h}$ on the Arizona Radio Observatory (ARO) in 2018 to obtain molecular gas mass measurements of MaNGA galaxies via the ${ }^{12} \mathrm{CO}(1-0)$ transition. After that first successful set of observations, MASCOT was granted another $1200 \mathrm{~h}$ with the ARO. A large survey for molecular gas in galaxies with existing IFU data represents a uniquely powerful tool for addressing a host of science questions and opens new avenues for molecular gas studies by drawing targets from the current generation of SDSS surveys.

The MASCOT survey complements other recent spatially resolved observational programmes in the community. The Extragalactic Database for Galaxy Evolution (EDGE) survey targeted 177 infraredbright Calar Alto Integral Field Area (CALIFA) survey galaxies using the Combined Array for Millimeter-wave Astronomy (CARMA). The survey targeted the ${ }^{12} \mathrm{CO}(1-0)$ line and its ${ }^{13} \mathrm{CO}$ isotopologue, providing spatially resolved $\mathrm{CO}$ maps with a resolution corresponding to size scales of $0.5-2 \mathrm{kpc}$ matched to the CALIFA observations (Bolatto et al. 2017). The maps have a half-power field of view (FOV) with a radius of $\sim 50$ arcsec, covering the galaxies out to 


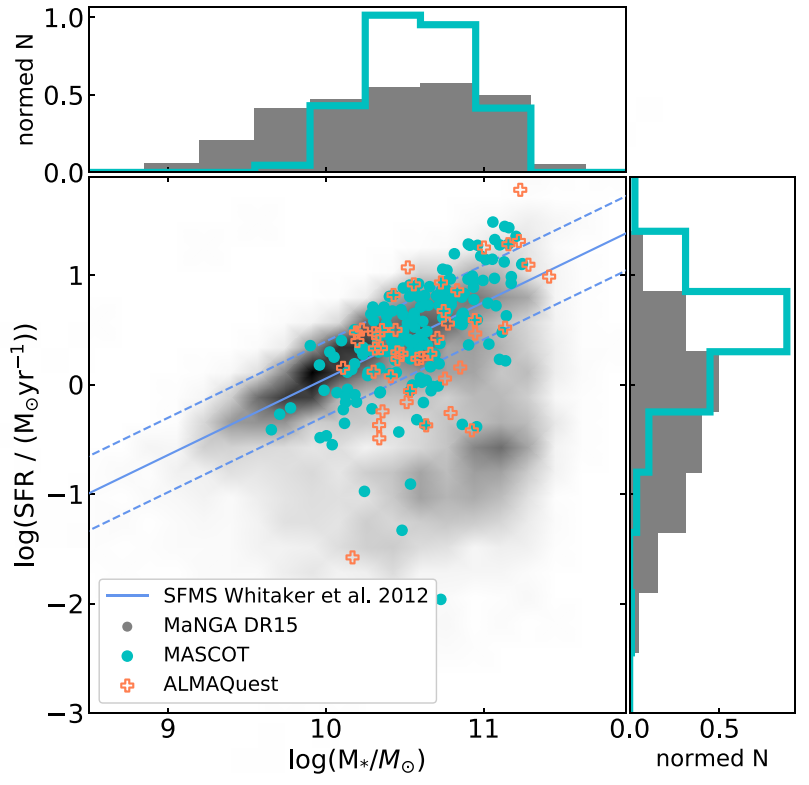

Figure 2. Distribution of the MASCOT targets in the SFR- $M_{*}$ plane (cyan circles). We also show the distribution of the DR15 MaNGA sample (grey density map) and the distribution of targets from the complementary ALMaQUEST sample (orange crosses). Additionally, we show the SFMS as derived by Whitaker et al. (2012) and its associated scatter of \pm 0.34 dex (dashed lines). Most MASCOT sources lie above the SFMS owing to the selection process for this first part of the MASCOT survey. The ALMaQUEST sources nicely complement the MASCOT sample in sampling sources on and below the SFMS. We also show the normalized distributions of the stellar masses $M_{*}$ and SFR above and next to the main panel. For the normalization, each bin displays the bin's raw count divided by the total number of counts and the bin width.

approximately two to three effective radii $\left(R_{\text {eff }}\right)$. This data set enables studies of the relationships between molecular gas and its kinematics, stellar mass, star formation rate, metallicity, and dust extinction on kiloparsec scales (Utomo et al. 2017; Colombo et al. 2018; Leung et al. 2018; Levy et al. 2018; Dey et al. 2019; Levy et al. 2019; Barrera-Ballesteros et al. 2020, 2021). These observations are currently being complemented by ${ }^{12} \mathrm{CO}(2-1)$ measurements of CALIFA galaxies using the APEX $12 \mathrm{~m}$ submillimetre telescope (Güsten et al. 2006). Compared with MASCOT, the CALIFA-APEX observations target galaxies at lower redshift $(0.005<z<0.03)$ and provide less coverage. The first set of combined CARMA and APEX observations of CALIFA galaxies comprises 472 sources (Colombo et al. 2020). The APEX CO(2-1) beam covers the galaxies roughly out to $1 R_{\text {eff. }}$ By dividing the sample into galaxies that are centrally quenched and star-forming galaxies, first results suggest that once star formation has been significantly reduced due to the consumption of molecular gas, changes in the SFE are what drive a galaxy deeper into the red sequence.

On the other hand, the Atacama Large Millimeter Array (ALMA)MaNGA QUEnching and STar formation (ALMaQUEST) survey is a programme with spatially resolved $12 \mathrm{CO}(1-0)$ measurements obtained with the ALMA for 46 galaxies from MaNGA DR15 (Lin et al. 2020). The aim of the ALMaQUEST survey is to investigate the dependence of star formation activity on the cold molecular gas content at kiloparsec scales in nearby galaxies and it targets not only main-sequence galaxies, but also starburst and green valley galaxies (Lin et al. 2019b; Ellison et al. 2020a,b; Lin et al. 2020; Ellison et al. 2021a,b). The survey spatially resolves the $\mathrm{CO}(1-0)$ line on scales matching the MaNGA resolution and its field of view is $\sim 50$ arcsec, very similar to the MASCOT observations. The ALMaQUEST survey is very complementary to MASCOT and we include their spatially integrated $\mathrm{CO}$ measurements in this paper (for details, see Section 3.8). Together, the EDGE-CALIFA and ALMaQUEST surveys provide complementary strengths for studying gas and star formation with supporting optical IFU data on kiloparsec scales in the nearby Universe.

In this paper, we present the first data release of the MASCOT survey of molecular gas mass measurements for 187 galaxies selected from the MaNGA survey. The paper is structured as follows. In Section 2, we summarize the available MaNGA data products and additional ancillary data for the galaxies. In Section 3, we present details on the MASCOT survey, including the sample selection, details on the observations with the ARO, data reduction, and analysis. Section 4 investigates some first global and resolved relations between the molecular gas mass measurements and the MaNGA-derived galaxy properties, and in Section 5 we conclude. For the derived quantities, we assume a flat Lambda cold dark matter $(\Lambda \mathrm{CDM})$ cosmology with $H_{0}=70 \mathrm{~km} \mathrm{~s}^{-1} \mathrm{Mpc}^{-1}, \Omega_{\mathrm{m}}$ $=0.3$, and $\Omega_{\Lambda}=0.7$. We furthermore use the redshifts published in the NASA-Sloan Atlas (NSA) catalogue ${ }^{1}$ (Blanton et al. 2011).

\section{SDSS MANGA}

\subsection{Survey description}

MaNGA is a 2D spectroscopic survey and is part of the SDSS-IV. MaNGA (Yan et al. 2016a) uses the Baryon Oscillation Spectroscopic Survey (BOSS) spectrographs (Gunn et al. 2006; Smee et al. 2013) with $R \sim 2000$ to take IFU observations of each galaxy in the 3600$10000 \AA$ range. Fibres are arranged into hexagonal bundles. The bundles have sizes that range from 19 to 127 fibres, depending on the apparent size of the target galaxy (which corresponds to diameters ranging between 12 and 32 arcsec). This leads to an average footprint of 400-500 $\mathrm{arcsec}^{2}$ per IFU. The fibres have a size of 2 arcsec aperture ( 2.5 arcsec separation between fibre centres), which corresponds to $\sim 2 \mathrm{kpc}$ at $z \sim 0.05$, although with dithering the effective sampling improves to 1.4 arcsec (see also Wylezalek et al. 2018). The current data release DR15 (Aguado et al. 2019) contains 4688 MaNGA galaxies (including ancillary targets and $\sim 65$ repeat observations). MaNGA has observed 10010 galaxies at $z \lesssim 0.15$ and with stellar masses $>10^{9} \mathrm{M}_{\odot}$, and the entire data set will become public as part of DR17 (currently scheduled for December 2021).

\subsection{MaNGA data products}

The data are first fed through the MaNGA Data Reduction Pipeline (DRP), which produces sky-subtracted spectrophotometrically calibrated spectra and rectified 3D data cubes (Wylezalek et al. 2018). These combine individual dithered observations (for details on MaNGA data reduction, see Law et al. 2016) with a spatial pixel scale of $0.5 \operatorname{arcsec}$ pixel $^{-1}$. The median spatial resolution of the MaNGA data is 2.54 arcsec full width at half-maximum (FWHM), while the median spectral resolution is $\sim 72 \mathrm{~km} \mathrm{~s}^{-1}$ (Law et al. 2016, 2021).

The MaNGA Data Analysis Pipeline (DAP; Belfiore et al. 2019; Westfall et al. 2019) is a project-led software package and is used

${ }^{1}$ http://nsatlas.org 
to analyse the data products produced by the MaNGA DRP. The analysis results of the DAP provide the collaboration and public with survey-level quantities, such as spectral indices, kinematics, and emission-line properties for 21 different emission lines. To make these calculations, the DAP first fits the stellar continuum using the penalized pixel-fitting method (pPXF; Cappellari \& Emsellem 2004; Cappellari 2017) and then performs a second fitting stage for the emission lines, which optimizes simultaneously the continuum and the emission lines, which are added as templates in a pPXF call. For example, for DR15, the DAP provides spatially stacked spectra, stellar kinematics ( $\mathrm{V}$ and $\sigma$ ) derived on a set of Voronoi-binned spectra, spaxel-based nebular emission-line properties including fluxes, equivalent widths, and kinematics ( $\mathrm{V}$ and $\sigma$ ), and spectral indices from absorption lines (e.g. $\mathrm{H} \delta$ ) and bandhead (e.g. D4000) measurements.

In this paper, we also make use of the Value Added Catalog of 'MaNGA Pipe3D' (Sanchez et al. 2018), which is based on measurements performed with the Pipe3D pipeline (Sánchez et al. 2016). The pipeline is designed to fit the continuum with stellar population models and to measure the nebular emission lines of IFS data. The stellar population library uses the Salpeter (1955) initial mass function. The stellar mass is then obtained based on the best-fitting stellar population model. The stellar population model spectra are then subtracted from the original cube, allowing the analysis of the ionized gas emission lines (Sánchez et al. 2016). For example, the star formation rate was derived by using the $\mathrm{H} \alpha$ luminosities for all the spaxels with detected ionized gas following the conversion given by Kennicutt (1998a) as well as from the single stellar population (SSP) fits computing the amount of stellar mass formed in the last $32 \mathrm{Myr}^{2}$ The value-added products include both a set of data cubes, containing spatially resolved stellar population properties, star formation histories, emission-line fluxes, and stellar absorption line indices derived using the Pipe3D pipeline, and a catalogue with one entry per galaxy, comprising the integrated properties of those galaxies (e.g. stellar mass and SFR), and the characteristic values (e.g. oxygen abundance at the effective radius, all including the associated uncertainties). ${ }^{3}$ In this paper, we make use of the integrated stellar masses and SSP-based SFRs.

We also point out to the reader that a number of additional 'value-added catalogues' have been publicly released by the SDSS collaboration. These catalogues have been created by MaNGA collaborators and are distributed through the SDSS Science Archive Server. In particular, we point out the 'HI-MaNGA Data Release 1' catalogue, which presents the first catalogue of H I $(21 \mathrm{~cm}$ neutral hydrogen) follow-up for MaNGA galaxies (Masters et al. 2019).

In this work, we furthermore make use of Marvin, a tool specifically designed to visualize and analyse MaNGA data. It is developed and maintained by the MaNGA team. Among other tools, Marvin allows the user to access reduced MaNGA data cubes locally, remotely, or via a web interface, access and visualize data analysis products and perform powerful queries on data and metadata. ${ }^{4}$

\footnotetext{
${ }^{2}$ Link to the data model of the Pipe3D catalogue: https://data.sdss.org/data model/files/MANGA_PIPE3D/MANGADRP_VER/

PIPE3D_VER/manga.Pipe3D.html

${ }^{3}$ Link to the Pipe3D Value Added Catalogs: https://www.sdss.org/dr14/mang a/manga-data/manga-pipe3d-value-added-catalog/

${ }^{4}$ See also the Marvin Documentation: https://sdss-marvin.readthedocs.io/en/ latest/
}

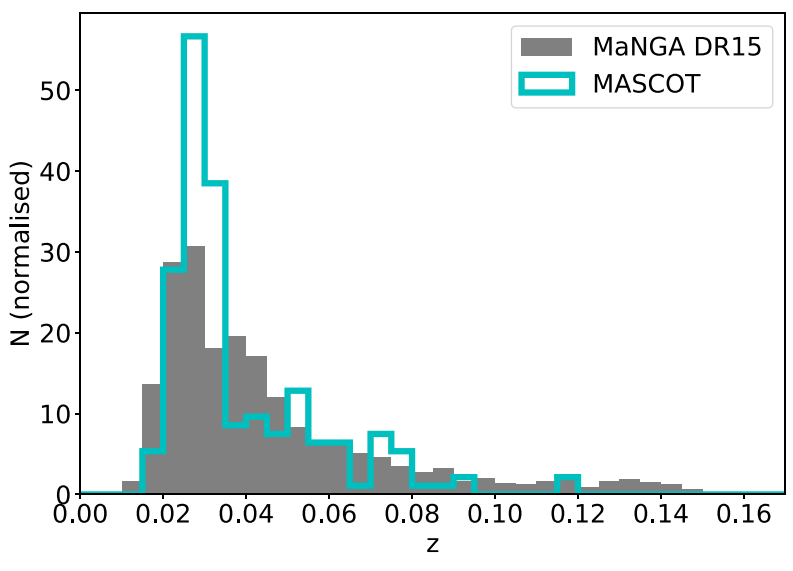

Figure 3. Normalized redshift distribution of the MASCOT sources in comparison with the MaNGA sample (DR15). For the normalization, each bin displays the bin's raw count divided by the total number of counts and the bin width.

Details about Marvin are described in Cherinka et al. (2019) and on the Marvin website.

\section{MASCOT SAMPLE AND OBSERVATIONS}

\subsection{Survey description}

The MASCOT sample is being assembled over the course of two programmes at the ARO as part of the MASCOT 1.0 and MASCOT 2.0 programmes (PI: Wylezalek). As part of the agreement for transferring ownership of the ALMA prototype antenna to the University of Arizona to install it on Kitt Peak as a telescope of the ARO, ESO distributed to its user community a total of $3600 \mathrm{~h}$ of observing time on the ARO telescopes from 2015 to $2020 .{ }^{5}$ It was decided that this time should be dedicated to large public surveys with an important legacy value. MASCOT was chosen to be one of these surveys.

The first part of the survey, MASCOT 1.0, was awarded $200 \mathrm{~h}$ of observing time in 2018 and the survey targeted 34 sources selected from the MaNGA survey. For the sample selection, we used the then available Data Release 14 (DR14; Abolfathi et al. 2018), which contained 2778 galaxies at $0.01<z<0.15$ with a mean $z \sim 0.05$.

The second part of the survey, MASCOT 2.0, was awarded $1200 \mathrm{~h}$ spread over four semesters in 2019 and 2020 (P103-P106). Due to the COVID-related shutdown of the telescope in 2020, the timeline of the execution of the observations has been significantly delayed. Sources have been selected from the current MaNGA data release DR15.

At the time of writing of this paper, observations for 153 sources have been completed as part of MASCOT 2.0, resulting in a total number of observed MASCOT targets of 187 . The distribution of the observed objects on the sky and in redshift space is shown in Figs 1 and 3 , respectively.

\subsection{Sample selection}

The aim of this survey is to provide molecular gas measurements for galaxies with existing IFU data. All targets are therefore drawn from

\footnotetext{
${ }^{5}$ http://www.eso.org/sci/activities/call_for_public_surveys.html
} 
the sample of targets with existing observations from the MaNGA survey. The current MaNGA data release at the time of the start of the MASCOT 1.0 programme in early 2018 consisted of $\sim 3000$ members, but the survey continued to observe $\sim 1600$ galaxies/year and public data releases happened on a yearly basis. As a result, the target list for MASCOT 2.0 has evolved and will continue to evolve over the remaining part of the survey, as MaNGA releases its complete and final data set (see Section 2.1).

We select our targets from MaNGA sources with available SFR measurements and we estimate the expected $\mathrm{CO}(1-0)$ luminosity from the total SFR by assuming the SFR $-L_{\mathrm{CO}(1-0)}^{\prime}$ scaling relation obtained by Cicone et al. (2017; shown in fig. 8 in their paper) for the ALLSMOG and COLD GASS samples of local star-forming galaxies (Saintonge et al. 2011; Cicone et al. 2017). We then derive the expected $\mathrm{CO}(1-0)$ integrated flux and the expected $\mathrm{CO}(1-0)$ peak flux density assuming a total line width of $200 \mathrm{~km} \mathrm{~s}^{-1}$. These estimates were carried out to prioritize the first set of sources to be observed within MASCOT. Since we carried out observations spread over the entire year, our sample selection is independent of any right ascension (RA) constraints.

To test the (at that time unknown) telescope efficiency, in 2018, we chose to start the survey with the brightest sources with an expected $\mathrm{CO}(1-0)$ peak flux density $>75 \mathrm{mJy}$, corresponding to a lower limit in molecular gas mass of $\log \left(M_{\mathrm{H}_{2}} / \mathrm{M}_{\odot}\right) \sim 8.9$ for a galaxy with a redshift of $z=0.02$ (which corresponds to the mean redshift of our sample; see Fig. 3). This selection ensured that we could either detect the $\mathrm{CO}(1-0)$ emission line in these targets at a signal-to-noise ratio $(\mathrm{S} / \mathrm{N})>3$ or infer valuable upper limits on their molecular gas content. We integrated until the CO line was detected, or until we reached a $1-\sigma_{\text {rms }}$ sensitivity of $0.5 \mathrm{mK}$ in $\delta v=50 \mathrm{~km} \mathrm{~s}^{-1}$ wide channels, corresponding to $\sim 13 \mathrm{mJy}$. That selection resulted in a sample of star-forming galaxies located with a mean of $\sim 0.15 \mathrm{dex}$ above the star formation main sequence (SFMS).

The number of detections has exceeded our expectations given the previous estimates of the sensitivity of the receiver. Furthermore, the telescope has been upgraded with a new spectrometer (see Section 3.3) since the start of the MASCOT programme in 2018. We have therefore adjusted our targeting strategy. To reduce the bias in selecting primarily star-forming galaxies, we have continued the survey drawing from the remaining MaNGA targets with an expected $\mathrm{CO}(1-0)$ peak flux density $>15 \mathrm{mJy}$ and adjusted our target $1-\sigma_{\mathrm{rms}}$ sensitivity to $0.25 \mathrm{mK}$. In Fig. 2, we show the distribution of the MASCOT sample in the SFR- $M_{*}$ plane in comparison with the full MaNGA sample, where we use the stellar masses and SSP-derived SFR from the Pipe3D catalogue. The current sample of 187 galaxies is located with a median of $\sim 0.07$ dex above the SFMS.

\subsection{Observations}

Observations are carried out with the new $12 \mathrm{~m}$ millimetre singledish telescope at the ARO. At the typical redshifts of the MaNGA sample $(z \sim 0.025)$, the $\mathrm{CO}(1-0)$ line is conveniently redshifted to $112 \mathrm{GHz}$, where the atmospheric opacity is improved relative to $115 \mathrm{GHz}$. We used the $3 \mathrm{~mm}$ receiver on the $12 \mathrm{~m} \mathrm{ARO}$ antenna (equivalent to ALMA band 3, 84-116 GHz). The first part of the MASCOT survey (MASCOT 1.0) was carried out with the millimetre autocorrelator (MAC) backend on the ARO $12 \mathrm{~m}$ antenna, which provided a bandwidth of $800 \mathrm{MHz}\left(600 \mathrm{MHz}\right.$ usable $\simeq 1600 \mathrm{~km} \mathrm{~s}^{-1}$ at $112 \mathrm{GHz}$ ). In August 2018, the new ARO Wideband Spectrometer (AROWS) backend was successfully commissioned on the $12 \mathrm{~m}$ Telescope. AROWS offers an increased total bandwidth of $4000 \mathrm{MHz}$, sampling $>5000 \mathrm{~km} \mathrm{~s}^{-1}$ around the $\mathrm{CO}(1-0)$ line and allowing for an improved baseline subtraction, especially for sources with broad $\mathrm{CO}(1-0)$ lines. The second part of the MASCOT survey (MASCOT 2.0) has been carried out with the AROWS backend, and so will be forthcoming observations. All observations are also still simultaneously recorded with the MAC, as a backup. Both the MAC and AROWS give a velocity resolution of $\sim 1 \mathrm{~km} \mathrm{~s}^{-1}$. Since our sources are 'high-velocity' sources, i.e. galaxies with $z>0.02$, we tuned the telescope to the different rest frequencies and set that rest frequency to $0.0 \mathrm{~km} \mathrm{~s}^{-1}$ for all sources.

Observations are carried out in fixed observing blocks. The atmospheric conditions varied greatly, with the precipitable water vapour (PWV) ranging between 2 and $20 \mathrm{~mm}$, with a mean of $\sim 5 \mathrm{~mm}$. Targets for this first data release were chosen and prioritized based on their expected peak $\mathrm{CO}(1-0)$ flux density as described in Section 3.2 as well as on their observability. For example, we prioritized targets with low $(<2)$ airmass and avoided pointing towards the wind direction. We made 'real-time' decisions on the targets by performing on-the-fly data reductions of the observations, and observations were stopped once the target was detected at a significance of $5 \sigma$ or when we reached a root mean square (rms) $\left(\sigma_{\mathrm{rms}}\right)$ of $0.5 \mathrm{mK}(0.25 \mathrm{mK}$; see Section 3.2$)$ per $50 \mathrm{~km} \mathrm{~s}^{-1}$ channel, corresponding to $\sim 13 \mathrm{mJy}(\sim 7 \mathrm{mJy})-$ whichever came first. In good observing conditions and for bright sources, conditions were met within $1 \mathrm{~h}$, but typical exposure times ranged between $\sim 2$ and $3 \mathrm{~h}$.

\subsection{Data reduction}

The data are reduced with the CLASS software. ${ }^{6}$ All scans are visually examined and unusually noisy scans, scans with distorted baselines or anomalies, are discarded. We then manually set a generous velocity window and estimate the baseline in each scan through a first-order fit to the continuum outside of that velocity window. If the $\mathrm{CO}$ line is undetected or very weak, we set the velocity window to $[-300$, $300] \mathrm{km} \mathrm{s}^{-1}$. The scans are then averaged and saved as a fits file.

These spectra are still in units of the observed source antenna temperature corrected for atmospheric attenuation, radiative loss, and rearward scattering and spillover $T_{\mathrm{R}}^{*}{ }^{*}$ We therefore first convert to the main beam temperature $T_{\mathrm{mb}}$ using $T_{\mathrm{mb}}=T_{\mathrm{R}}^{*} / \eta_{\mathrm{m}}^{*}$, where $\eta_{\mathrm{m}}^{*}$ is the corrected main beam efficiency. The flux density $f_{v}$ can then be derived from the Rayleigh-Jeans law such that

$f_{v}=5.097 \times 10^{-4} \frac{\mathrm{BWHM}^{2}}{\lambda^{2}} T_{\mathrm{mb}}$,

where $f_{v}$ is in janskys, $\lambda$ is the observing wavelength in centimetres, and BWHM (beamwidth at half maximum) is the beam size in arcseconds. The beam size at the frequency of our observations is 55 arcsec. $^{8}$ The typical uncertainty in the main beam efficiency is $\sim 3-4$ per cent.

\subsection{Spectral line fitting}

\subsubsection{Dynamical spectral binning}

We develop a customized spectral line fitting technique to measure the CO line fluxes and kinematic parameters. We use the baseline subtracted averaged spectra in units of jansky as described in

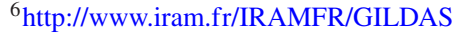

${ }^{7}$ See appendix C of the ARO $12 \mathrm{~m}$ manual: http://aro.as.arizona.edu/12_obs manual

${ }^{8}$ See table 3.2 of the ARO $12 \mathrm{~m}$ manual: http://aro.as.arizona.edu/12_obs_m anual
} 
Section 3.4. Our aim is to dynamically bin the spectra depending on the measured $\mathrm{S} / \mathrm{N}$ at a given bin width $\mathrm{d} v_{\text {bin }}$. This procedure utilizes non-parametric flux and velocity width measurements, which are described in more detail in Section 3.5.2.

We begin by fitting a single Gaussian profile to the spectrum at its native spectral resolution $\left(1-2 \mathrm{~km} \mathrm{~s}^{-1}\right.$ depending on the redshift of the source) and allow the velocity offset $\Delta v$ to range between -250 and $250 \mathrm{~km} \mathrm{~s}^{-1}$ and the velocity dispersion to range between 50 and $500 \mathrm{~km} \mathrm{~s}^{-1}$. We then measure the cumulative flux

$S(v)=\int_{-\infty}^{v} f_{v}\left(v^{\prime}\right) \mathrm{d} v^{\prime}$,

and the total line flux is given by $S(\infty)$. In practice, we use the interval $[-3000,3000] \mathrm{km} \mathrm{s}^{-1}$ in the rest frame of the galaxy for the integration.

We then determine $v_{05}$ such that $S\left(v_{05}\right)=0.05 \times S(\infty)$ and $v_{95}$ such that $S\left(v_{95}\right)=0.95 \times S(\infty)$. These are the velocities at which 5 percent and 95 percent of the total flux $S(\infty)$ are reached, respectively. We use these velocities to determine the width of the fitted line $W_{90}$ using $W_{90}=v_{95}-v_{05}$. We measure the flux $S$ of the line from the data (i.e. not the Gaussian model) within $W_{90}$ and refer to it as $S_{\mathrm{CO} \text {,data }}$ (see Section 3.5.2). For the $S_{\mathrm{CO} \text {,data }}$, we include the flux bins in which $v_{05}$ and $v_{95}$ fall.

We then determine the uncertainty $\epsilon$ on the flux by first determining the rms noise of the spectra. We measure the standard deviation of the noise per spectral channel, $\sigma_{\text {rms }}$, outside the spectral window of $[-250,250] \mathrm{km} \mathrm{s}^{-1}$ (with respect to the $0 \mathrm{~km} \mathrm{~s}^{-1}$ rest frequency; see Section 3.3) and then compute

$\epsilon=\frac{\sigma_{\mathrm{rms}} W_{90}}{\sqrt{N}}$,

where $N$ is the number of channels within $W_{90}$, which can be determined by calculating $W_{90} / \mathrm{d} v_{\text {bin }}$.

If $\mathrm{S} / \mathrm{N}=S(\infty) / \epsilon$ is determined to be $<20$, we spectrally bin the spectrum to a resolution of $\mathrm{d} v_{\text {bin }}=10 \mathrm{~km} \mathrm{~s}^{-1}$ and repeat the described procedure. We repeat this process of increasing $\mathrm{d} v_{\text {bin }}$ in steps of $10 \mathrm{~km} \mathrm{~s}^{-1}$ until the line is measured with an $\mathrm{S} / \mathrm{N}>20$ or until we rebin the spectrum to a maximum $\mathrm{d} v_{\text {bin }}=50 \mathrm{~km} \mathrm{~s}^{-1}$. This procedure allows us to retain a better spectral sampling for the bright sources within our sample.

We provide the binned and unbinned spectra as part of the Supporting Information of this paper, as well as on the MASCOT website. ${ }^{9}$

\subsubsection{CO flux and CO luminosity measurements}

Once the spectral binning (to a maximum of $50 \mathrm{~km} \mathrm{~s}^{-1}$ ) has been optimized, we proceed with measuring the $\mathrm{CO}$ line flux. A single Gaussian is often insufficient for describing the profile of the $\mathrm{CO}(1-$ 0) line. This is particularly true when secondary broad components characteristic of potentially outflowing gas or broad or double-peaked $\mathrm{CO}(1-0)$ emission in the case of rotating gas discs is contributing significantly to the emission-line profile. To evaluate the prevalence of additional kinematic components, we therefore allow for two Gaussian components to be fitted to the $\mathrm{CO}$ line. The fitting procedure uses a least-squares regression to return best-fitting parameters for the single-Gaussian and double-Gaussian model. The $\chi^{2}$ value is then used to evaluate the goodness of the fit.

${ }^{9}$ https://www.staff.ari.uni-heidelberg.de/dwylezalek/mascot.html
In cases where the spectral shape of an emission line is close to Gaussian, then the calculation of best-fitting parameters from a single Gaussian fit (i.e. velocity dispersion, FWHM, amplitude) is sufficient to describe its kinematic properties. This is not the case when multiple Gaussians are needed to describe the line profile (see also Wylezalek et al. 2020). We therefore calculate non-parametric values based on the percentages of the total integrated flux (see e.g. Liu et al. 2013). We compute the $S(v)$ based on the best model fit (see equation). We then compute the line-of-sight velocity $v_{\text {med }}$ where $S\left(v_{\text {med }}\right)=0.5 \times S(\infty)$; i.e., this is the velocity that bisects the total area underneath the emission-line profile. Because the fitting is performed in the rest frame of the galaxy as determined by its stellar component, $v_{\text {med }}$ is measured relative to the rest frame (see also Wylezalek et al. 2020). We use the $W_{90}$ parameter to parametrize the velocity width of the $\mathrm{CO}$ line. $W_{90}$ refers to the velocity width that encloses 90 percent of the total flux. We determine $v_{05}$ and $v_{95}$ and then calculate $W_{90}$ using $W_{90}=v_{95}-v_{05}$. The advantage of using $W_{90}$ over the Gaussian velocity dispersion is that it quasiindependent of the underlying model used to fit the profile. We also compute $W_{50}=v_{75}-v_{25}$ for easier comparison with other works and enhancing the legacy value of our work.

We then compute the $\mathrm{CO}(1-0)$ line flux in three different ways: (i) by integrating the spectrum data within $v_{05}$ and $v_{95}$, yielding $S_{\mathrm{CO} \text {,data }}$; (ii) by integrating the model fit within $v_{05}$ and $v_{95}$, yielding $S_{\mathrm{CO} \text {,model }}$; and (iii) by integrating the model fit within $[-3000,3000] \mathrm{km} \mathrm{s}^{-1}$, yielding $S_{\mathrm{CO} \text {,tot }}$.

The measured line fluxes agree well with one another, generally within 2-10 per cent, while the uncertainty on the flux measurements due to the noise in the data ranges between 10 per cent and 20 per cent. For the remaining analysis in this paper, we therefore use $S_{\mathrm{CO} \text {,model }}$. We refer to this measurement from now on as $S_{\mathrm{CO}}$ and report it in Table 1 .

We compute the uncertainty $\epsilon$ on the flux measurement following equation (3) and determine the $S / N$ of the emission line by computing $\mathrm{S} / \mathrm{N}=S_{\mathrm{CO}} / \epsilon$. We also compute the $\mathrm{S} / \mathrm{N}$ of the emission-line peak, $\mathrm{S} / \mathrm{N}_{\text {peak }}$, by determining the peak flux density within the velocity window set by $W_{90}, f_{\text {peak }}$, and computing $\mathrm{S} / \mathrm{N}_{\text {peak }}=f_{\text {peak }} / \sigma_{\text {rms. }}$. We consider a source to be detected if $\mathrm{S} / \mathrm{N}>3$ or $S / \mathrm{N}_{\text {peak }}>3$. Based on this definition, we detect 162 out of 187 sources. If we had chosen an $\mathrm{S} / \mathrm{N}$ cut of 4, 132 sources would be considered detected.

For the 162 detected sources, we compute the corresponding $\mathrm{CO}(1-0)$ luminosity $L_{\mathrm{CO}}$ using the equation

$L_{\mathrm{CO}}=4 \pi D_{\mathrm{L}}^{2} S_{\mathrm{CO}}$,

where $D_{\mathrm{L}}$ is the luminosity distance.

In case of a non-detection, we report the $3 \sigma$ upper limits, where $\sigma$ is determined following equation (3) assuming a constant $W_{90}$ of 200 or $300 \mathrm{~km} \mathrm{~s}^{-1}$ for galaxies with $M_{*}$ lower or greater than $10^{10} \mathrm{M}_{\odot}$, respectively (see also Saintonge et al. 2017).

Table 1 reports the CO line luminosities (or upper limits for the non-detections) in units of $\mathrm{L}_{\odot}$ as well as $\mathrm{K} \mathrm{km} \mathrm{s}^{-1} \mathrm{pc}^{2}$, the kinematic parameters of the line fit (the velocity width $W_{90}$, the line-of-sight velocity $v_{\text {med }}$, as well as the velocity width $\left.W_{50}\right)$, the $\mathrm{S} / \mathrm{N}$, and the $\sigma_{\text {rms }}$ of the spectrum.

\section{6 $\mathrm{H}_{2}$ masses}

The ${ }^{12} \mathrm{CO}$ molecule is a commonly used tracer for the cold molecular gas content in galaxies of which most is in the form of $\mathrm{H}_{2}$. Since the $\mathrm{H}_{2}$ molecule lacks a permanent electric dipole moment, cold $\mathrm{H}_{2}$ is not directly observable but the total molecular gas mass can be 


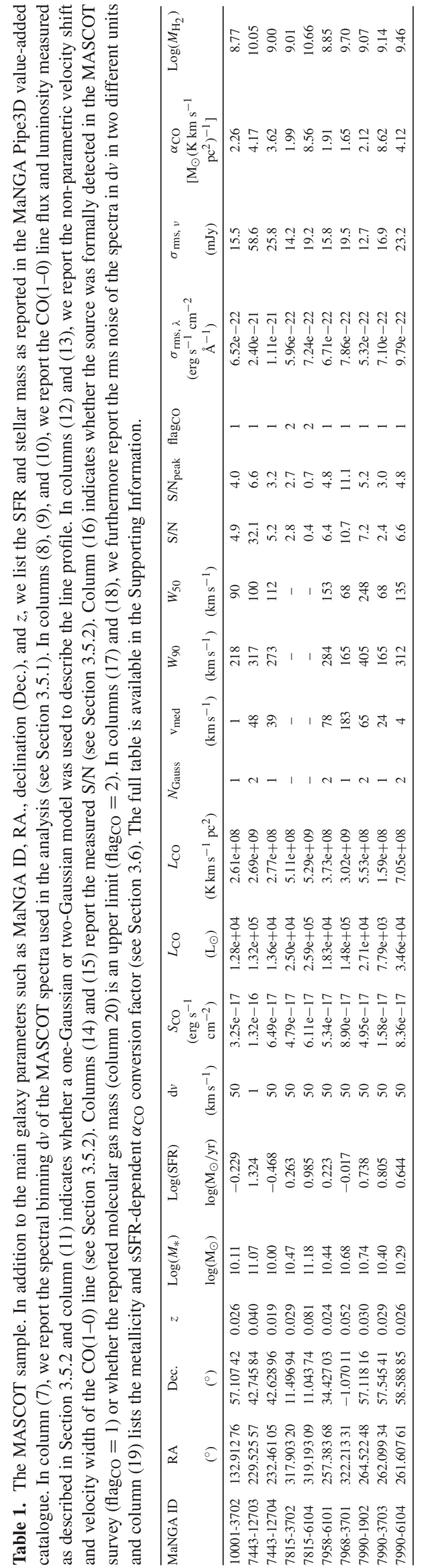

estimated following the empirical relation

$M_{\mathrm{H}_{2}}=\alpha_{\mathrm{CO}} L_{\mathrm{CO}(1-0)}^{\prime}$,

where $M_{\mathrm{H}_{2}}$ is in units of $\mathrm{M}_{\odot}$ and $L_{\mathrm{CO}(1-0)}^{\prime}$ is in units of $\mathrm{K} \mathrm{km} \mathrm{s}^{-1} \mathrm{pc}^{2}$. $\alpha_{\mathrm{CO}}$, the CO-to- $\mathrm{H}_{2}$ conversion factor, can be considered a mass-tolight ratio. The empirical value of $4.36 \mathrm{M}_{\odot}\left(\mathrm{K} \mathrm{km} \mathrm{s}^{-1} \mathrm{pc}^{2}\right)^{-1}$, referred to as the Galactic conversion factor, has been determined from observations of the Milky Way and nearby star-forming galaxies. While this Galactic conversion factor is often applied to other galaxies, as well, it is well known that the conversion factor is dependent on many parameters, mainly the SFR and gas-phase metallicity. For example, $\mathrm{UV}$ radiation from massive stars destroys $\mathrm{CO}$ to a cloud depth of a few $A_{V}$, such that the Galactic conversion factor would underestimate the true molecular hydrogen content (see Bolatto et al. 2013 for a review).

Accurso et al. (2017) recently used results from the xCOLD GASS survey (Saintonge et al. 2017) combined with Herschel observations to carry out a thorough Bayesian analysis revealing that only two parameters, gas-phase metallicity $\log (\mathrm{O} / \mathrm{H})$ and offset from the SFMS $\Delta(\mathrm{MS})$, are needed to robustly parametrize changes in the $L_{[\mathrm{C} \mathrm{II]}} / L_{\mathrm{CO}(1-0)}$ ratio. They use their parametrization of that ratio, alongside radiative transfer modelling, to present a novel conversion function for $\alpha_{\mathrm{CO}}$ :

$$
\begin{array}{r}
\log \alpha_{\mathrm{CO}}( \pm 0.165 \text { dex })=15.623-1.732[12+\log (\mathrm{O} / \mathrm{H})] \\
+0.051 \log \Delta(\mathrm{MS})
\end{array}
$$

where the distance off the SFMS is defined as

$\Delta(\mathrm{MS})=\frac{\mathrm{sSFR}_{\text {measured }}}{\mathrm{sSFR}_{\mathrm{ms}}\left(z, M_{*}\right)}$

and where the analytical definition of the SFMS by Whitaker et al. (2012) is used:

$$
\begin{array}{r}
\log \left(\mathrm{sSFR}_{\mathrm{ms}}\left(z, M_{*}\right)\right)=-1.12+1.14 z-0.19 z^{2}-(0.3+0.13 z) \\
\times\left(\log M_{*}-10.5\right)\left(\mathrm{Gyr}^{-1}\right) .
\end{array}
$$

We make use of the relation presented by Accurso et al. (2017) to derive a metallicity- and sSFR-dependent $\alpha_{\mathrm{CO}}$. We use the $\log (\mathrm{O} / \mathrm{H})$ oxygen abundance at the effective radius derived using the Maiolino et al. (2008) calibrator provided in the MaNGA Pipe3D catalogue ('OH_Re_fit_M08'), as well as the SFR and stellar masses from the MaNGA Pipe3D catalogue ('log_SFR_ssp', 'log_mass'). In Accurso et al. (2017), the gas-phase metallicity from Pettini \& Pagel (2004) is used in equation (6), which has been shown to have a constant offset of 0.05 from the Maiolino et al. (2008) calibrator in the mass range of our galaxies (Sánchez et al. 2017). We therefore correct the Pipe3D $\log (\mathrm{O} / \mathrm{H})$ oxygen abundance by +0.05 before calculating $\alpha_{\text {CO }}$. For 27 galaxies, the $\log (\mathrm{O} / \mathrm{H})$ abundance is not reported in the Pipe3D catalogue due to insufficient data quality. We assign a $\alpha_{\mathrm{CO}}$ to those galaxies corresponding to the median value of $2.68 \mathrm{M}_{\odot}$ $\left(\mathrm{K} \mathrm{km} \mathrm{s}^{-1} \mathrm{pc}^{2}\right)^{-1}$ of our remaining galaxy sample.

We then calculate the molecular gas masses following equation (5). For sources undetected in the MASCOT CO(1-0) observations, we report upper limits based on the $3 \sigma$ upper limits on their $\mathrm{CO}(1-0)$ luminosity (see Table 1).

\subsection{Cross-match with xCOLD GASS and ALLSMOG}

The xCOLD GASS survey is a large legacy survey providing a census of molecular gas in the local Universe by having obtained $\mathrm{CO}(1-0)$ observations of 532 galaxies with the IRAM $30 \mathrm{~m}$ telescope (Saintonge et al. 2017). The sample was mass selected in the redshift interval $0.01<z<0.05$ from the SDSS spectroscopic sample. 

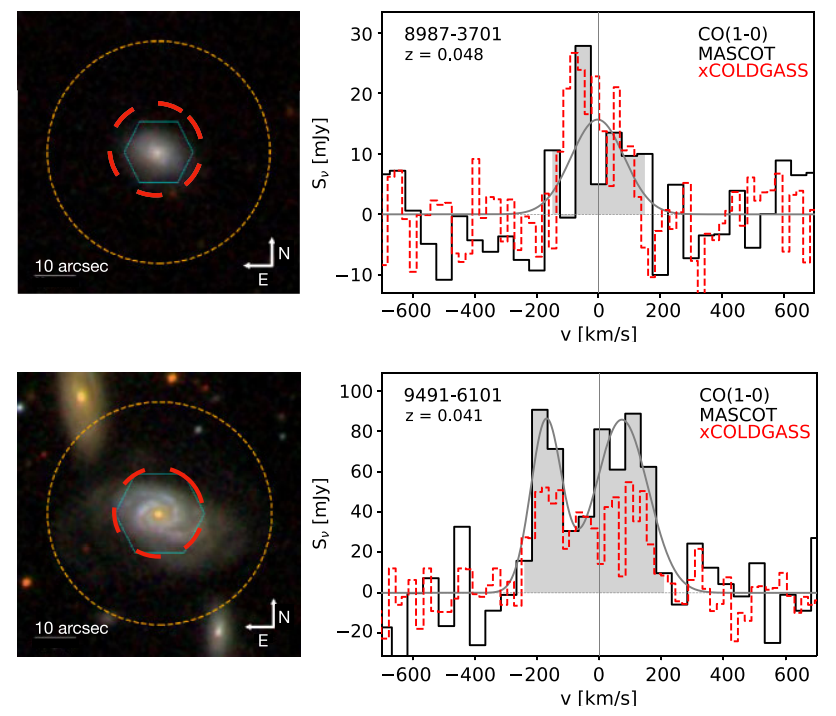

Figure 4. Comparison of $x$ COLD GASS and MASCOT observations for two targets the surveys have in common. We show the SDSS three-colour image, showing the MaNGA footprint (purple hexagon), the ARO beam (orange dashed circle), and the IRAM $30 \mathrm{~m}$ beam of the xCOLD GASS observations (red dashed circle). The spectra on the right show the MASCOT CO(1-0) line spectrum (solid black line) and the xCOLD GASS spectrum (red dashed line). The area of the MASCOT spectrum between $v_{05}$ and $v_{95}$ is coloured in light grey.

We therefore cross-match the MASCOT catalogue with the XCOLD GASS catalogue to identify any overlapping observations.

There are three sources observed within both the xCOLD GASS and MASCOT surveys. Fig. 4 shows a comparison of two spectra obtained within both projects. The IRAM beam size at the frequency of the $\mathrm{CO}(1-0)$ observations is $22 \mathrm{arcsec}$ in contrast to the ARO beam size of $55 \mathrm{arcsec}$ (see Fig. 4). For MASCOT source 89873701 (xCOLD GASS ID: 32619), the IRAM beam extends beyond $3 R_{\text {eff }}$ of the galaxy $\left(R_{\text {eff }}=3.1 \mathrm{arcsec}\right)$, while the effective radius of MASCOT source 9491-6101 (xCOLD GASS ID: 31775 ) is $R_{\text {eff }}=$ 5.6 arcsec and therefore only covered by the IRAM beam out to $\sim 2 R_{\text {eff }}$. This difference is reflected in the respective spectra obtained by MASCOT and xCOLD GASS (Fig. 4). While the spectra for 8987-3701 are very similar and their measured CO luminosities are in good agreement (within 15 per cent and within uncertainties), the xCOLD GASS observations of 9491-6101 seem to miss a significant amount of flux (CO luminosities are within 40 per cent and are not within uncertainties), even though Saintonge et al. (2017) do apply an aperture correction to their $\mathrm{CO}$ flux measurements.

The median $r$-band effective radius of the xCOLD GASS sample is 4.7 arcsec, very close to the median $r$-band effective radius of the MaNGA survey of 5.4 arcsec. This means that 9491-6101 (xCOLD GASS ID: 31775 ) is not an outlier with an unusually large size in the xCOLD GASS survey but representative of the entire xCOLD GASS sample. This implies that despite aperture corrections, smaller beam molecular gas surveys may miss a significant amount of molecular gas. Therefore, $\mathrm{CO}$ surveys with beam sizes covering galaxies out to several effective radii such as the MASCOT survey are better suited for assessing the total molecular gas content of these low-redshift galaxies.

We also cross-match the MASCOT sample with the ALLSMOG survey (Cicone et al. 2017) but do not find any overlap in sources.

\subsection{ALMaQUEST survey}

The ALMaQUEST survey is a programme with spatially resolved $12 \mathrm{CO}(1-0)$ measurements obtained with ALMA for 46 galaxies from MaNGA DR15 (Lin et al. 2020). The aim of the ALMaQUEST survey is to investigate the dependence of star formation activity on the cold molecular gas content at kiloparsec scales in nearby galaxies. While this survey spatially resolves the $\mathrm{CO}(1-0)$ line on scales matching the MaNGA resolution, its field of view is $\sim 50$ arcsec, very similar to the MASCOT observations. The ALMaQUEST and MASCOT surveys have four sources in common (ID: 8952-6104, 8950-12705, 8450-6102, and 8655-3701), whose derived molecular gas masses agree well: $\log \left(M_{\mathrm{H}_{2}, \mathrm{MASCOT}} / \mathrm{M}_{\odot}\right)$ $\left.=9.2 / 9.2 / 9.2 / 10.2 \operatorname{and} \log \left(M_{\mathrm{H}_{2}, \text { ALMaQUEST }} / \mathrm{M}_{\odot}\right)=9.1 / 9.4 / 9.2 / 10.4\right)$. The ALMaQUEST survey is thus well complementary to the MASCOT survey (see also Fig. 2).

\section{RESULTS}

\subsection{Global relations}

In Fig. 5, we show the distribution of galaxies in the SFR- $M_{*}$ plane. We complement the MASCOT sample with results from the ALMaQUEST survey, which mainly complements the MASCOT survey in the green valley. While we show the MASCOT nondetections in Fig. 5 for completeness, the following analysis is limited to the 162 MASCOT-detected sources for which we can measure $M_{\mathrm{H}_{2}}$. Together with the 43 additional detected sources from the ALMaQUEST survey, the following analysis focuses on a total sample size of 205 sources.

For consistency, and similar to Fig. 2, for both the MASCOT and ALMaQUEST samples, we use the SFR and $M_{*}$ measurements provided by the Pipe3D catalogue. We note that this differs from what is presented and shown in table 3 and fig. 3 in the ALMaQUEST paper by Lin et al. (2020), where the SFR is computed based on considering star-forming spaxels (as per Baldwin, Phillips \& Telervich (BPT) classification) within $1.5 R_{\text {eff }}$.

We also remind the reader that the ALMaQUEST molecular gas mass measurements were measured by summing the $\mathrm{CO}(1-0)$ flux over the areas within the MaNGA bundles, while the MASCOT beam covers generally a larger area (beam size: 55 arcsec). This implies that the ALMaQUEST molecular gas fractions may be lower when directly compared with the MASCOT molecular gas fractions. In the case of an underestimation of the $\mathrm{CO}(1-0)$ luminosity by 60 per cent (as is the case for source 9491-6101 in comparison with the XCOLD GASS survey; see Section 3.7), $\log \left(f_{\text {mol }}\right)$ would be underestimated by 0.3 .

The upper left-hand panel of Fig. 5 is colour coded by the molecular gas fraction defined as $f_{\mathrm{mol}}=M_{\mathrm{H}_{2}} / M_{*}$. For the MASCOT sources, this fraction is directly computed using the $M_{\mathrm{H}_{2}}$ measurements described in Section 3.6 and $M_{*}$ from the Pipe3D catalogue. We measure a median molecular gas mass fraction for the MASCOT survey of $f_{\text {mol }}=0.052$. For the ALMaQUEST sources, we use the molecular gas masses reported in table 3 in Lin et al. (2020) and the $M_{*}$ from the Pipe3D catalogue to compute $f_{\text {mol }}$.

The left-hand panel of Fig. 5 illustrates that $f_{\text {mol }}$ is roughly constant along the SFMS and sharply drops below the SFMS. We observe a significant correlation with specific star formation rate $\left(\mathrm{sSFR}=\mathrm{SFR} / M_{*}\right)$ with a Spearman rank correlation coefficient of $r_{\text {corr }}=0.45$ and a $P$-value of $1 \times 10^{-11}$. The correlation with SFR alone is more moderate, with $r_{\text {corr }}=0.3$ and a $P$-value of $1 \times 10^{-6}$, while we do not observe a significant correlation with stellar mass $\left(r_{\text {corr }}=0, P\right.$-value of 0.5$)$. 

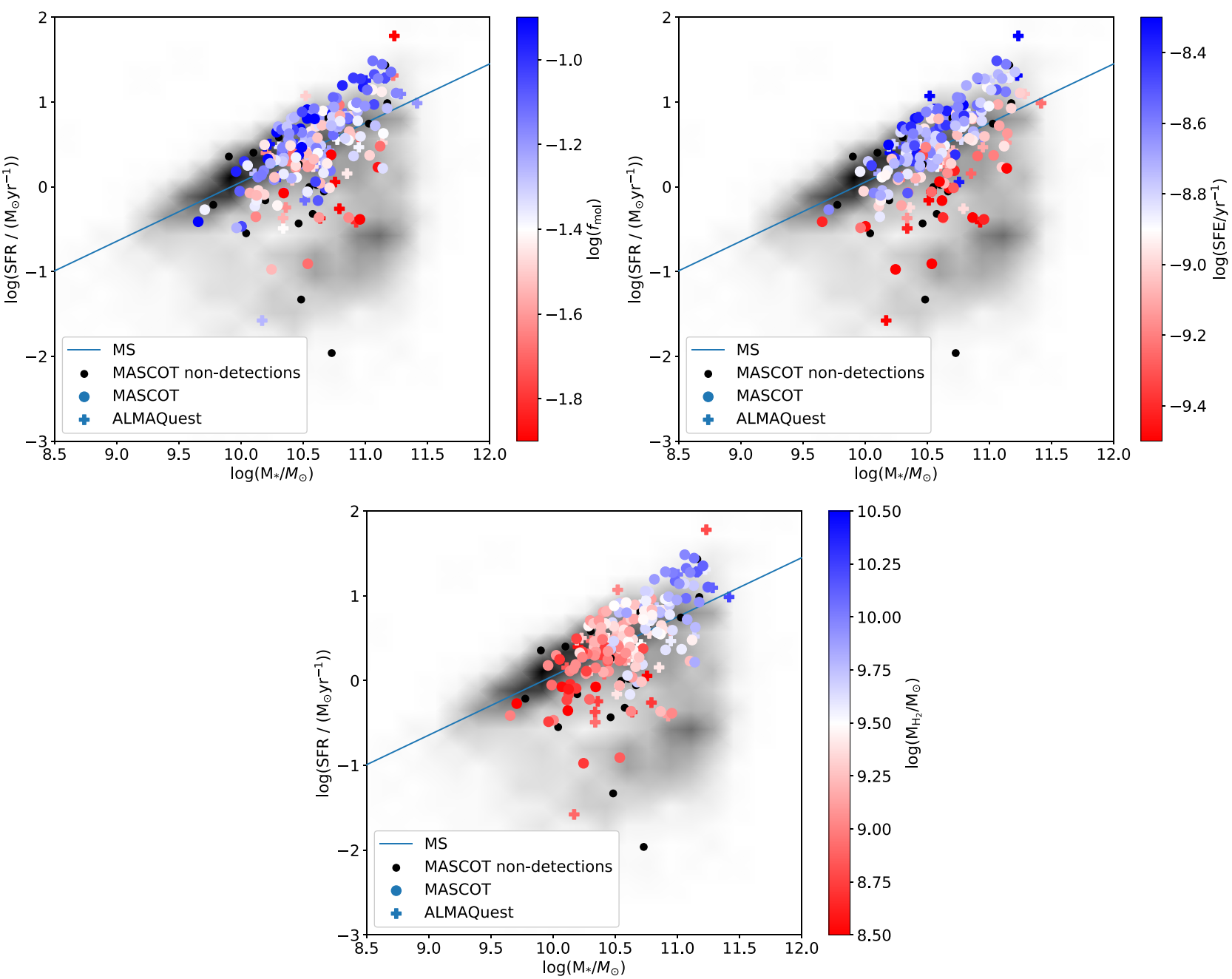

Figure 5. We show the distribution of sources in the SFR- $M_{*}$ diagrams, colour coded by the molecular gas mass fraction $f_{\text {mol }}=M_{\mathrm{H}_{2}} / M_{*}$ (upper left-hand panel), the $\mathrm{SFE}=\mathrm{SFR} / M_{\mathrm{H}_{2}}$ (upper right-hand panel), and the molecular gas mass $M_{\mathrm{H}_{2}}$ (lower panel). For reference, we also show the distribution of the DR15 MaNGA sample in the SFR- $M_{*}$ plane, as well as the SFMS as derived by Whitaker et al. (2012). SFRs and stellar masses are both taken from the MaNGA Pipe3D catalogue for all sources, while the molecular gas masses are derived from the 55 arcsec ARO beam for the MASCOT sources and from the MaNGA footprint for the ALMaQUEST sources.

Similar trends are observed when we investigate how the molecular $\mathrm{SFE}=\mathrm{SFR} / M_{\mathrm{H}_{2}}$ varies across the SFMS. The MASCOT median $\mathrm{SFE}$ is $\log \left(\mathrm{SFE} / \mathrm{yr}^{-1}\right)=-8.7$. The inverse of the SFE is the 'depletion time', $\tau_{\text {dep }}$, indicating how much time is necessary to convert all the available molecular gas into stars at the current SFR. We find that the depletion time is roughly constant along the SFMS, as well, with depletion times dropping sharply below the SFMS. The correlation between both SFE and SSFR and SFE and SFR are significant, with $r_{\text {corr }}=0.68, P$-value of $10^{-29}$ and $r_{\text {corr }}=0.47, P$ value of $10^{-13}$, respectively. SFE seems to be independent of stellar mass, though $\left(r_{\text {corr }}=0, P\right.$-value of 0.2$)$.

In contrast to the first two panels of Fig. 5, the lower panel shows that the molecular mass $M_{\mathrm{H}_{2}}$ varies strongly along the SFMS, with significant correlations of $M_{\mathrm{H}_{2}}$ with both SFR and $M_{*}\left(r_{\text {corr }} \sim 0.7 / 0.7\right.$, $P$-value $=10^{-31} / P$-value $=10^{-36}$, respectively). The median molecular gas mass in the MASCOT sample is $\log \left(M_{\mathrm{H}_{2}} / \mathrm{M}_{\odot}\right)=9.3$.

The observed relations are similar and consistent with other studies in the literature. For example, Colombo et al. (2020) carried out a large $\mathrm{CO}(1-0)$ survey of 472 galaxies selected from the CALIFA survey with the APEX telescope and the CARMA array. The CALIFA CO sample is of lower redshift than the MaNGA CO observations presented here. Furthermore, their $\mathrm{CO}$ observations are measured from within $\sim 1 R_{\text {eff }}$ and therefore only measure the centrally available molecular gas. Despite these differences, the observed relations between SFR, $M_{\mathrm{H}_{2}}, f_{\mathrm{mol}}$, and SFE are very similar to what we observe here. Similar conclusions were also made by Saintonge et al. (2017) with the xCOLD GASS survey. These observations imply that it is both the molecular gas mass fraction and the SFE that determine a galaxy's position in the SFR- $M_{*}$ plane.

We note that since we are using a prescription for $\alpha_{\mathrm{CO}}$ that is dependent on $\Delta(\mathrm{MS})$, the derived quantities $f_{\mathrm{mol}}$ and SFE are dependent on $M_{*}$ and SFR in a non-trivial way. We therefore repeat the earlier analysis for a constant $\alpha_{\mathrm{CO}}$ and do not find significant changes in the trends reported in this section. But even without the added dependence of $\alpha_{\mathrm{CO}}$ on $\Delta(\mathrm{MS})$, derived quantities such as $\mathrm{SFE}=\mathrm{SFR} / M_{\mathrm{H}_{2}}$ and $\mathrm{sSFR}=\mathrm{sSFR}=\mathrm{SFR} / M_{*}$ are not independent and therefore some form of correlation is expected. Cicone et al. (2017) found that $L_{\mathrm{CO}}$ (from which $M_{\mathrm{H}_{2}}$ is derived) is strongly 


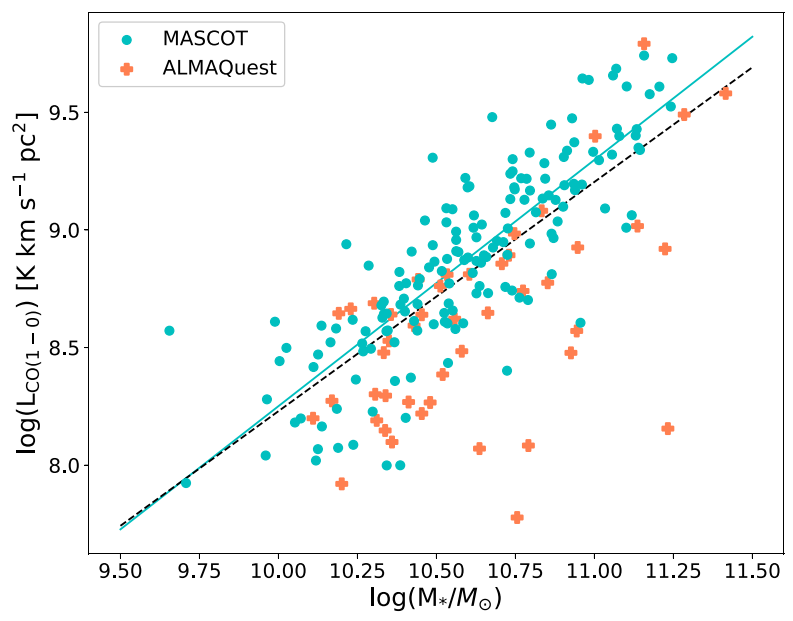

Figure 6. $\mathrm{CO}(1-0)$ line luminosity as a function of $M_{*}$. The cyan solid line indicates the best-fitting linear relation obtained for the MASCOT data only $(y=-2.21+1.05 x)$, while the black dashed line indicates the bestfitting relation for the combined MASCOT and ALMaQUEST samples $(y=$ $-1.51+0.97 x)$

correlated with $M_{*}$ and suggested that that this relation may be so tight and linear because the luminosity of optically thick low$J$ CO transitions is an excellent tracer of the dynamical mass in star-forming galaxies, assuming that in this class of objects the bulk of $\mathrm{CO}$ probes molecular clouds in virial motions. We test this correlation with our observations and find a similarly tight correlation as Cicone et al. (2017) $\left(r_{\text {corr }}=0.84, P\right.$-value $=10^{-45}$; see Fig. 6$)$. We note the slight offset between the ALMaQUEST data points and the MASCOT data points in Fig. 6. As stated earlier, this is very likely due to the fact that the ALMaQUEST molecular gas mass measurements were measured by summing the $\mathrm{CO}(1-0)$ flux over the areas within the MaNGA bundles, while the MASCOT beam covers generally a larger area. Therefore, the total ALMaQUEST CO measurements tend to be slightly underestimated, similar to our findings with the xCOLD GASS galaxies (see Section 3.7). Nevertheless, the bestfitting regression parameters and correlation coefficients presented in Fig. 6 are in very good agreement with the Cicone et al. (2017) results.

\subsection{Resolved relations}

While the preceding section reassuringly confirms previous results, the main aim and true strength of the MASCOT survey are to investigate how the molecular gas mass content relates to spatially resolved galaxy parameters. Although an in-depth analysis of the resolved relations will be presented in upcoming papers in this series (Bertemes et al. in preparation), we present here an example of how the MASCOT survey is and will be used in investigating global $\mathrm{CO}$ measurements together with the spatially resolved nature of the MaNGA observations.

In Fig. 7, we show how the molecular gas mass fraction, $f_{\text {mol }}$, relates to the stellar age gradient $\alpha_{\text {age,LW. }}{ }^{10}$ The stellar age gradient describes the slope of the gradient of the luminosity-weighted log age of the stellar population within a galactocentric distance of $0.5-2.0 R_{\text {eff }}$.

\footnotetext{
${ }^{10}$ Although we use $\alpha$ to refer to the $\mathrm{CO}$-to- $\mathrm{H}_{2}$ conversion factor, we also use $\alpha$ - albeit with a different index - to refer to the stellar age gradient to be consistent with the notation in the Pipe3D catalogue.
}

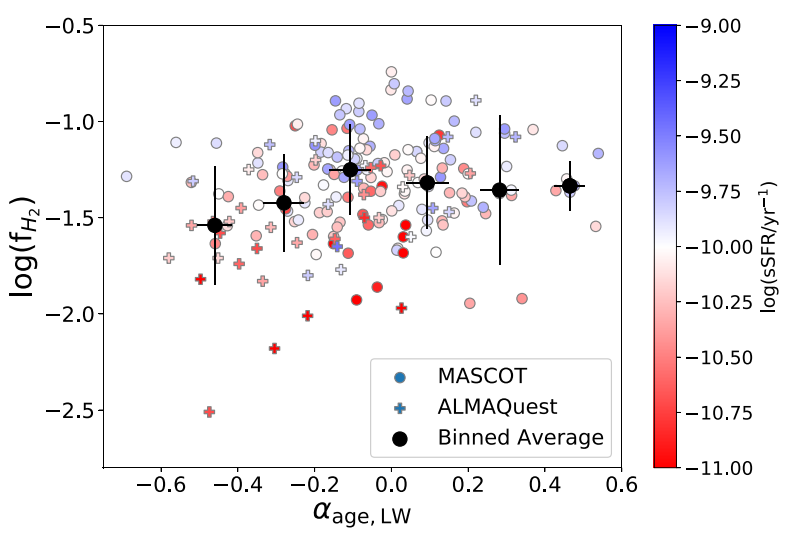

Figure 7. The molecular gas mass fraction $f_{\text {mol }}$ as a function of stellar age gradient $\alpha_{\text {age,LW }}$. Galaxies with lower $f_{\text {mol }}$ tend to show older stellar populations close to the galactic centre, while the opposite is true for galaxies with higher $f_{\text {mol }}$, potentially a signature for inside-out quenching being the dominant quenching mechanism in the MASCOT MaNGA galaxies.

This measurement is provided as part of the Pipe3D catalogue. While the Pipe3D catalogue also reports mass-weighted ages, luminosityweighted ages are more sensitive to small fractions of recent generations of stars (which contribute significantly in luminosity but not in mass); the mass-weighted age is more representative of the average epoch when the bulk of the stars in a galaxy formed (see e.g. Pasquali et al. 2010). Since we are investigating potential quenching, or recent star formation triggering effects, the luminosityweighted ages are more meaningful for this analysis. When $\alpha$ is positive, the stellar populations are getting older as we move away from the galactic centre. When $\alpha$ is negative, it indicates that the stellar populations are getting younger as we move away from the galactic centre. Despite the large scatter, we observe a weak positive correlation between $\alpha_{\text {age, LW }}$ and $f_{\text {mol }}\left(r_{\text {corr }}=0.2, P\right.$-value $\left.=4 \times 10^{-3}\right)$. The correlation is less weak when only considering galaxies with a negative $\alpha_{\text {age } \mathrm{LW}}\left(r_{\text {corr }}=0.3, P\right.$-value $\left.=2 \times 10^{-4}\right)$. This means that galaxies with high molecular gas mass fractions tend to host younger stellar populations in their centres than they do at larger distances, while galaxies with low molecular gas mass fractions tend to be centrally quenched. This observation suggests that star formation quenching in these galaxies happens primarily inside out and that a lower $\mathrm{H}_{2}$ gas fraction seems to be a precursor of quenching.

Evidence for inside-out quenching being the dominant quenching mode has been suggested by other works (Lin et al. 2017; Bluck et al. 2020; Breda et al. 2020; Brownson et al. 2020). Such analyses have been in particular possible due to the emergence of large IFU galaxy surveys. For example, Lin et al. (2019a) classify MaNGA galaxies into inside-out and outside-in quenching types. They base their classification on the strength and spatial distribution of quenched areas that are defined by two non-parametric parameters, quiescence and its concentration, traced by regions with low EW ( $\mathrm{H} \alpha$ ) (for details and the definitions of these parameters, see Lin et al. 2019a). Additionally, they classify the galaxies based on their environment into satellites and centrals. They find that the fraction of inside-out quenching is systematically greater than that of outside-in quenching, suggesting that inside-out quenching is the dominant quenching mode in all environments.

AGN feedback and morphological quenching are potential mechanisms that may suppress the star formation and that may drive the features of the inside-out quenching, but which one dominates under 

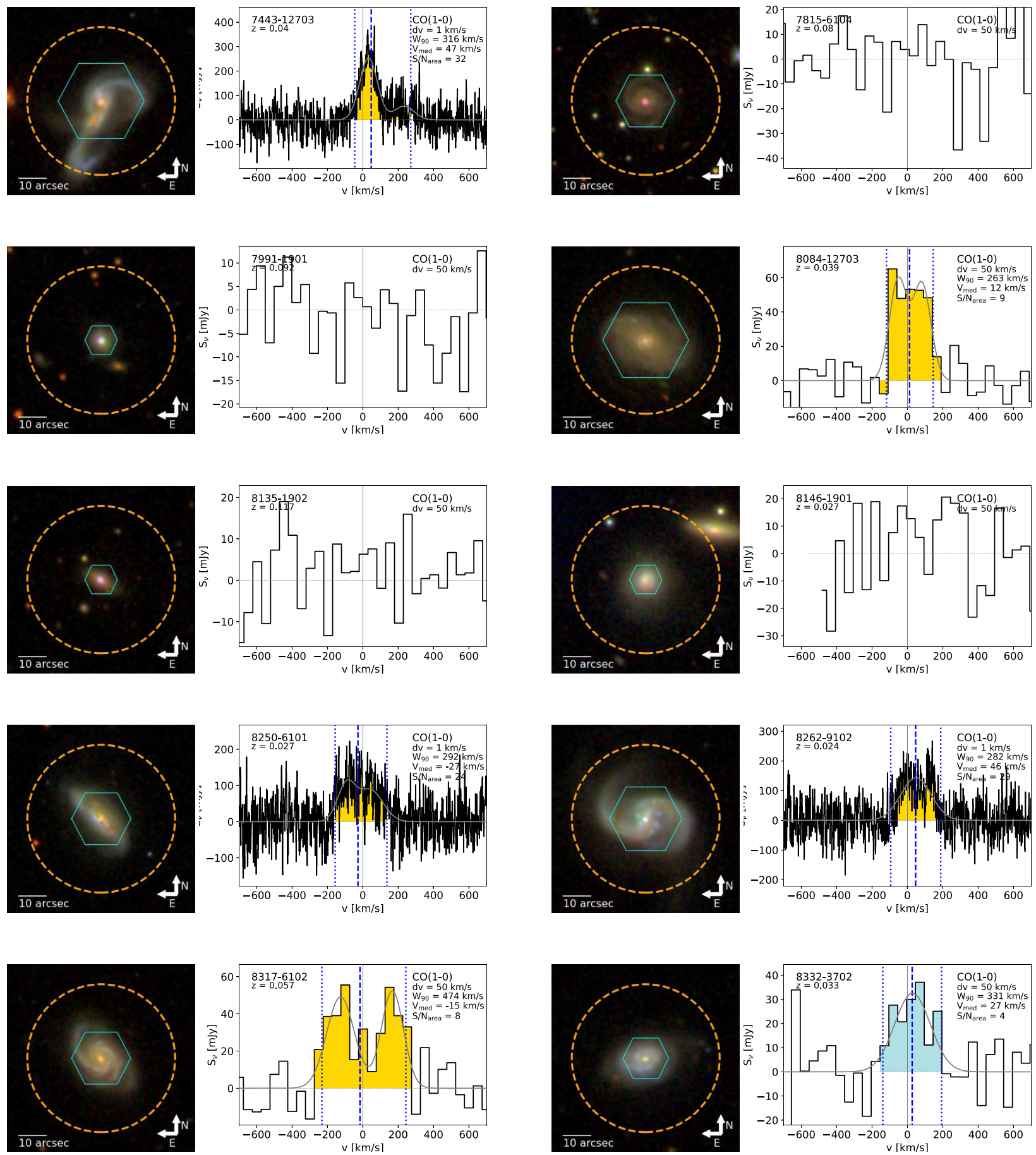

Figure 8. Left: SDSS three-colour image, also showing the MaNGA footprint (purple hexagon) and the ARO beam (orange dashed circle). Right: the MASCOT $\mathrm{CO}(1-0)$ line spectrum (solid black line). In cases where the spectrum was spectrally binned, the spectrum in native spectral resolution is shown in light grey. The final spectral resolution $\mathrm{d} v$ is reported in the plot. When the $\mathrm{CO}(1-0)$ line is detected, the model fit is shown as the grey solid line and the line-of-sight velocity $v_{\text {med }}$ is indicated by the blue dashed line. Both $v_{05}$ and $v_{95}$, which are used for the $W_{90}$ determination, are indicated by the dotted blue lines. For sources detected at an $\mathrm{S} / \mathrm{N}>5$, the part of the spectrum between $v_{05}$ and $v_{95}$ that is used for the data-based line flux measurement $S_{\mathrm{CO} \text {,data }}$ is coloured in yellow. For tentative detections with $3<\mathrm{S} / \mathrm{N}<5$, that part is coloured in light blue. The image cut-outs and figures are also available as part of the Supporting Information.

what circumstances is still an open point of debate (Lin et al. 2017, 2019a; Ellison et al. 2021c). The current size of the MASCOT sample and the parameter space it covers do not allow us yet to investigate this question in detail. But as the MASCOT sample continues to grow, we will address these topics in the forthcoming papers in this series. 


\section{CONCLUSION}

In this paper, we have presented the first data release of the MASCOT survey, which provides $\mathrm{CO}(1-0)$ measurements for galaxies that have already been observed as part of the SDSS-IV MaNGA survey. Observations are carried out with the $12 \mathrm{~m}$ antenna at the Arizona Radio Observatory. We summarize the main points of this paper in the following:

(i) This first data release presents $\mathrm{CO}(1-0)$ observations for 187 MaNGA galaxies. These galaxies are mostly located on and above the SFMS and upcoming MASCOT observations are targeting primarily MaNGA galaxies on and below the SFMS.

(ii) We develop a customized spectral line fitting technique to allow for a dynamic spectral binning of the spectra (from native binning up to spectral bins of $50 \mathrm{~km} \mathrm{~s}^{-1}$ ) depending on the $\mathrm{S} / \mathrm{N}$ of the detection. To measure CO line fluxes, we allow secondary Gaussian components to account for broad, asymmetric line profiles and/or double-Gaussian features. We use non-parametric measurements to compute the flux and kinematics (velocity shift and velocity width) of the $\mathrm{CO}(1-0)$ emission line.

(iii) We use a metallicity- and sSFR-dependent $\alpha_{\mathrm{CO}}$ conversion factor to compute the total molecular gas mass within the beam size of the observations, 55 arcsec.

(iv) The molecular gas mass fraction $f_{\mathrm{mol}}=M_{\mathrm{H}_{2}} / M_{*}$ varies strongly across the SFMS and correlates most strongly with sSFR $\left(r_{\text {corr }}=0.45\right)$ and SFR $\left(r_{\text {corr }}=0.3\right)$. Similar trends are observed for the star formation efficiency, SFE $=\mathrm{SFR} / M_{\mathrm{H}_{2}}$. SFE correlates strongly with sSFR $\left(r_{\text {corr }}=0.68\right)$ and SFR $\left(r_{\text {corr }}=0.47\right)$ but is mostly independent of stellar mass. These observations imply that it is both the molecular gas mass fraction and the SFE that determine a galaxy's position in the SFR $-M_{*}$ plane, confirming previous results.

(v) When investigating the MASCOT CO measurements in the context of the spatially resolved information from the supporting MaNGA data, we find that $f_{\text {mol }}$ weakly correlates with the luminosity-

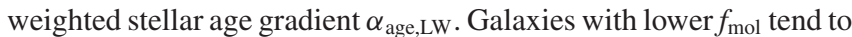
show older stellar populations close to the galactic centre, while the opposite is true for galaxies with higher $f_{\text {mol }}$, potentially a signature for inside-out quenching being the dominant quenching mechanism in the MASCOT MaNGA galaxies.

The MASCOT survey is a large programme and this data release only represents the first sets of observations of $\sim 800 \mathrm{~h}$ of observing time out of $1400 \mathrm{~h}$ total. Upcoming data releases will present results for galaxies primarily on and below the SFMS and the upcoming papers in this series (Bertemes et al., in preparation; Wylezalek et al., in preparation) are investigating in depth the relations between molecular gas mass properties and spatially resolved diagnostics from the MaNGA observations. With the current survey efficiency, we expect the final MASCOT sample to consist of a total of $\sim 250$ 300 galaxies.

\section{ACKNOWLEDGEMENTS}

The entire MASCOT team would like to warmly thank the staff at the Arizona Radio Observatory, in particular the operators of the $12 \mathrm{~m}$ Telescope, Clayton, Kevin, Mike, and Robert, for their continued support and help with the observations.

DW is supported by through the Emmy Noether Programme of the German Research Foundation. SC acknowledges the financial support from the State Agency for Research of the Spanish MCIU through the 'Center of Excellence Severo Ochoa' award to the Instituto de Astrofísica de Andalucía (SEV-2017-0709). MA acknowl- edges support from FONDECYT grant 1211951, 'CONICYT + PCI + INSTITUTO MAX PLANCK DE ASTRONOMIA MPG190030' and 'CONICYT+PCI + REDES 190194'. RM acknowledges support by the Science and Technology Facilities Council (STFC) and ERC Advanced Grant 695671 "QUENCH".

This research made use of Marvin, a core PYTHON package and web framework for MaNGA data, developed by Brian Cherinka, José Sánchez-Gallego, Brett Andrews, and Joel Brownstein (Cherinka et al. 2018) (http://sdss-marvin.readthedocs.io/en/stable/).

This project makes use of the MaNGA Pipe3D data products. We thank the IA-UNAM MaNGA team for creating this catalogue, and the ConaCyt-180125 project for supporting them.

Funding for the Sloan Digital Sky Survey-IV has been provided by the Alfred P. Sloan Foundation, the U.S. Department of Energy Office of Science, and the Participating Institutions.

SDSS-IV acknowledges support and resources from the Center for High Performance Computing at the University of Utah. The SDSS website is www.sdss.org.

SDSS-IV is managed by the Astrophysical Research Consortium for the Participating Institutions of the SDSS Collaboration including the Brazilian Participation Group, the Carnegie Institution for Science, Carnegie Mellon University, Center for Astrophysics | Harvard \& Smithsonian, the Chilean Participation Group, the French Participation Group, Instituto de Astrofísica de Canarias, the Johns Hopkins University, Kavli Institute for the Physics and Mathematics of the Universe (IPMU)/University of Tokyo, the Korean Participation Group, Lawrence Berkeley National Laboratory, Leibniz Institut für Astrophysik Potsdam (AIP), Max-Planck-Institut für Astronomie (MPIA Heidelberg), Max-Planck-Institut für Astrophysik (MPA Garching), Max-Planck-Institut für Extraterrestrische Physik (MPE), National Astronomical Observatories of China, New Mexico State University, New York University, University of Notre Dame, Observatário Nacional/MCTI, the Ohio State University, Pennsylvania State University, Shanghai Astronomical Observatory, United Kingdom Participation Group, Universidad Nacional Autónoma de México, University of Arizona, University of Colorado Boulder, University of Oxford, University of Portsmouth, University of Utah, University of Virginia, University of Washington, University of Wisconsin, Vanderbilt University, and Yale University.

\section{DATA AVAILABILITY}

The data underlying this article are available in the article and in its supplementary material. We also publish the data on the MASCOT website https://www.staff.ari.uni-heidelberg.de/dwylezalek/mascot. html.

\section{REFERENCES}

Abolfathi B. et al., 2018, ApJS, 235, 42

Accurso G. et al., 2017, MNRAS, 470, 4750

Aguado D. S. et al., 2019, ApJS, 240, 23

Barrera-Ballesteros J. K. et al., 2020, MNRAS, 492, 2651

Barrera-Ballesteros J. K. et al., 2021, MNRAS, 503, 3643

Belfiore F. et al., 2019, AJ, 158, 160

Blanton M. R., Kazin E., Muna D., Weaver B. A., Price-Whelan A., 2011, AJ, 142, 31

Blanton M. R. et al., 2017, AJ, 154, 28

Bluck A. F. L. et al., 2020, MNRAS, 499, 230

Bolatto A. D., Wolfire M., Leroy A. K., 2013, ARA\&A, 51, 207

Bolatto A. D. et al., 2017, ApJ, 846, 159

Bothwell M. S. et al., 2014, MNRAS, 445, 2599

Bouché N. et al., 2010, ApJ, 718, 1001 
Breda I. et al., 2020, A\&A, 635, A177

Brownson S., Belfiore F., Maiolino R., Lin L., Carniani S., 2020, MNRAS, 498, L66

Bundy K. et al., 2015, ApJ, 798, 7

Cappellari M., 2017, MNRAS, 466, 798

Cappellari M., Emsellem E., 2004, PASP, 116, 138

Cappellari M. et al., 2011, MNRAS, 413, 813

Cherinka B., Sánchez-Gallego J., Andrews B., Brownstein J., 2018, sdss/marvin: Marvin Beta, doi:10.5281/zenodo.596700, https://doi.org/ 10.5281/zenodo.596700

Cherinka B. et al., 2019, AJ, 158, 74

Cicone C. et al., 2017, A\&A, 604, A53

Colombo D. et al., 2018, MNRAS, 475, 1791

Colombo D. et al., 2020, A\&A, 644, A97

Combes F., Young L. M., Bureau M., 2007, MNRAS, 377, 1795

Croom S. M. et al., 2012, MNRAS, 421, 872

Dey B. et al., 2019, MNRAS, 488, 1926

Drory N. et al., 2015, AJ, 149, 77

Ellison S. L., Thorp M. D., Pan H.-A., Lin L., Scudder J. M., Bluck A. F. L., Sánchez S. F., Sargent M., 2020a, MNRAS, 492, 6027

Ellison S. L. et al., 2020b, MNRAS, 493, L39

Ellison S. L., Lin L., Thorp M. D., Pan H.-A., Scudder J. M., Sánchez S. F., Bluck A. F. L., Maiolino R., 2021a, MNRAS, 501, 4777

Ellison S. L., Lin L., Thorp M. D., Pan H.-A., Sánchez S. F., Bluck A. F. L., Belfiore F., 2021b, MNRAS, 502, L6

Ellison S. L. et al., 2021c, MNRAS, 505, L46

Gunn J. E. et al., 2006, AJ, 131, 2332

Güsten R., Nyman L. Å., Schilke P., Menten K., Cesarsky C., Booth R., 2006, A\&A, 454, L13

Harrison C. M., 2017, Nat. Astron., 1, 0165

Kennicutt R. C. Jr, 1998a, ARA\&A, 36, 189

Kennicutt R. C. Jr, 1998b, ApJ, 498, 541

Law D. R. et al., 2015, AJ, 150, 19

Law D. R. et al., 2016, AJ, 152, 83

Law D. R. et al., 2021, AJ, 161, 52

Leung G. Y. C. et al., 2018, MNRAS, 477, 254

Levy R. C. et al., 2018, ApJ, 860, 92

Levy R. C. et al., 2019, ApJ, 882, 84

Lilly S. J., Carollo C. M., Pipino A., Renzini A., Peng Y., 2013, ApJ, 772, 119

Lin L. et al., 2017, ApJ, 851, 18

Lin L. et al., 2019a, ApJ, 872, 50

Lin L. et al., 2019b, ApJ, 884, L33

Lin L. et al., 2020, ApJ, 903, 145

Liu G., Zakamska N. L., Greene J. E., Nesvadba N. P. H., Liu X., 2013, MNRAS, 436, 2576

Maiolino R. et al., 2008, A\&A, 488, 463

Masters K. L. et al., 2019, MNRAS, 488, 3396

Pasquali A., Gallazzi A., Fontanot F., van den Bosch F. C., De Lucia G., Mo H. J., Yang X., 2010, MNRAS, 407, 937
Péroux C., Howk J. C., 2020, ARA\&A, 58, 363

Pettini M., Pagel B. E. J., 2004, MNRAS, 348, L59

Rupke D., 2018, Galaxies, 6, 138

Saintonge A. et al., 2011, MNRAS, 415, 32

Saintonge A. et al., 2016, MNRAS, 462, 1749

Saintonge A. et al., 2017, ApJS, 233, 22

Salpeter E. E., 1955, ApJ, 121, 161

Sánchez S. F. et al., 2012, A\&A, 538, A8

Sánchez S. F. et al., 2016, Rev. Mex. Astron. Astrofis., 52, 21

Sánchez S. F. et al., 2017, MNRAS, 469, 2121

Sánchez S. F. et al., 2018, Rev. Mex. Astron. Astrofis., 54, 217

Sanders D. B., Mirabel I. F., 1985, ApJ, 298, L31

Smee S. A. et al., 2013, AJ, 146, 32

Solomon P. M., Downes D., Radford S. J. E., Barrett J. W., 1997, ApJ, 478, 144

Tumlinson J., Peeples M. S., Werk J. K., 2017, ARA\&A, 55, 389

Utomo D. et al., 2017, ApJ, 849, 26

Wake D. A. et al., 2017, AJ, 154, 86

Westfall K. B. et al., 2019, AJ, 158, 231

Whitaker K. E., van Dokkum P. G., Brammer G., Franx M., 2012, ApJ, 754, L29

Wylezalek D., Zakamska N. L., Greene J. E., Riffel R. A., Drory N., Andrews B. H., Merloni A., Thomas D., 2018, MNRAS, 474, 1499

Wylezalek D., Flores A. M., Zakamska N. L., Greene J. E., Riffel R. A., 2020, MNRAS, 492, 4680

Yan R. et al., 2016a, AJ, 151, 8

Yan R. et al., 2016b, AJ, 152, 197

Young L. M. et al., 2011, MNRAS, 414, 940

\section{SUPPORTING INFORMATION}

Supplementary data are available at MNRAS online.

Figure 8. Left: SDSS three-colour image, also showing the MaNGA footprint (purple hexagon) and the ARO beam (orange dashed circle). Right: the MASCOT CO(1-0) line spectrum (solid black line).

Table 1. The MASCOT sample. In addition to the main galaxy parameters such as MaNGA ID, RA, Dec., and $z$, we list the SFR and stellar mass as reported in the MaNGA Pipe3D Value Added Catalog.

Please note: Oxford University Press is not responsible for the content or functionality of any supporting materials supplied by the authors. Any queries (other than missing material) should be directed to the corresponding author for the article.

This paper has been typeset from a $\mathrm{T}_{\mathrm{E}} \mathrm{X} / \mathrm{L} \mathrm{T} \mathrm{E} \mathrm{X}$ file prepared by the author. 